\section{GENERAL RULES}

Actae Naturae publishes experimental articles and reviews, as well as articles on topical issues, short reviews, and reports on the subjects of basic and applied life sciences and biotechnology.

The journal is published by the Park Media publishing house in both Russian and English.

The journal Acta Naturae is on the list of the leading periodicals of the Higher Attestation Commission of the Russian Ministry of Education and Science

The editors of Actae Naturae ask of the authors that they follow certain guidelines listed below. Articles which fail to conform to these guidelines will be rejected without review. The editors will not consider articles whose results have already been published or are being considered by other publications.

The maximum length of a review, together with tables and references, cannot exceed 60,000 symbols (approximately 40 pages, A4 format, 1.5 spacing, Times New Roman font, size 12) and cannot contain more than 16 figures.

Experimental articles should not exceed 30,000 symbols (20 pages in A4 format, including tables and references). They should contain no more than ten figures. Lengthier articles can only be accepted with the preliminary consent of the editors.

A short report must include the study's rationale, experimental material, and conclusions. A short report should not exceed 12,000 symbols (8 pages in A4 format including no more than 12 references). It should contain no more than four figures.

The manuscript should be sent to the editors in electronic form: the text should be in Windows Microsoft Word 2003 format, and the figures should be in TIFF format with each image in a separate file. In a separate file there should be a translation in English of: the article's title, the names and initials of the authors, the full name of the scientific organization and its departmental affiliation, the abstract, the references, and figure captions.

\section{MANUSCRIPT FORMATTING}

The manuscript should be formatted in the following manner:

- Article title. Bold font. The title should not be too long or too short and must be informative. The title should not exceed 100 characters. It should reflect the major result, the essence, and uniqueness of the work, names and initials of the authors.

- The corresponding author, who will also be working with the proofs, should be marked with a footnote*.

- Full name of the scientific organization and its departmental affiliation. If there are two or more scientific organizations involved, they should be linked by digital superscripts with the authors' names. Abstract. The structure of the abstract should be very clear and must reflect the following: it should introduce the reader to the main issue and describe the experimental approach, the possibility of practical use, and the possibility of further research in the field. The average length of an abstract is 20 lines
(1,500 characters).

- Keywords $(3-6)$. These should include the field of research, methods, experimental subject, and the specifics of the work. List of abbreviations.

- INTRODUCTION

- EXPERIMENTAL PROCEDURES

- RESULTS AND DISCUSSION

- CONCLUSION

The organizations that funded the work should be listed at the end of this section with grant numbers in parenthesis.

\section{- REFERENCES}

The in-text references should be in brackets, such as [1].

\section{RECOMMENDATIONS ON THE TYPING \\ AND FORMATTING OF THE TEXT}

- We recommend the use of Microsoft Word 2003 for Windows text editing software.

- The Times New Roman font should be used. Standard font size is 12 .

- The space between the lines is 1.5 .

- Using more than one whole space between words is not recommended.

- We do not accept articles with automatic referencing; automatic word hyphenation; or automatic prohibition of hyphenation, listing, automatic indentation, etc.

- We recommend that tables be created using Word software options (Table $\rightarrow$ Insert Table) or MS Excel. Tables that were created manually (using lots of spaces without boxes) cannot be accepted.

- Initials and last names should always be separated by a whole space; for example, A. A. Ivanov.

- Throughout the text, all dates should appear in the "day.month.year" format, for example 02.05.1991, 26.12.1874, etc.

- There should be no periods after the title of the article, the authors' names, headings and subheadings, figure captions, units ( - second, g - gram, min minute, h- hour, d - day, deg - degree).

- Periods should be used after footnotes (including those in tables), table comments, abstracts, and abbreviations (mon. - months, y. - years, m. temp. melting temperature); however, they should not be used in subscripted indexes $\left(\mathrm{T}_{\mathrm{m}}\right.$ - melting temperature; $\mathrm{T}_{\mathrm{p.t}}$ - temperature of phase transition). One exception is mln - million, which should be used without a period.

- Decimal numbers should always contain a period and not a comma $(0.25$ and not 0,25$)$.

- The hyphen ("-") is surrounded by two whole spaces, while the "minus," "interval," or "chemical bond" symbols do not require a space.

- The only symbol used for multiplication is " $x$ "; the " $\times$ " symbol can only be used if it has a number to its right. The "." symbol is used for denoting complex compounds in chemical formulas and also noncovalent complexes (such as DNA·RNA, etc.).

- Formulas must use the letter of the Latin and Greek alphabets. 
- Latin genera and species' names should be in italics, while the taxa of higher orders should be in regular font.

- Gene names (except for yeast genes) should be italicized, while names of proteins should be in regular font.

- Names of nucleotides (A, T, G, C, U), amino acids (Arg, Ile, Val, etc.), and phosphonucleotides (ATP, AMP, etc.) should be written with Latin letters in regular font.

- Numeration of bases in nucleic acids and amino acid residues should not be hyphenated (T34, Ala89).

- When choosing units of measurement, SI units are to be used.

- Molecular mass should be in Daltons (Da, KDa, MDa).

- The number of nucleotide pairs should be abbreviated (bp, kbp).

- The number of amino acids should be abbreviated to aa.

- Biochemical terms, such as the names of enzymes, should conform to IUPAC standards.

- The number of term and name abbreviations in the text should be kept to a minimum.

- Repeating the same data in the text, tables, and graphs is not allowed.

\section{GUIDENESS FOR ILLUSTRATIONS}

- Figures should be supplied in separate files. Only TIFF is accepted.

- Figures should have a resolution of no less than 300 dpi for color and half-tone images and no less than 500 dpi.

- Files should not have any additional layers.

\section{REVIEW AND PREPARATION OF THE}

\section{MANUSCRIPT FOR PRINT AND PUBLICATION}

Articles are published on a first-come, first-served basis. The publication order is established by the date of acceptance of the article. The members of the editorial board have the right to recommend the expedited publishing of articles which are deemed to be a priority and have received good reviews.

Articles which have been received by the editorial board are assessed by the board members and then sent for external review, if needed. The choice of reviewers is up to the editorial board. The manuscript is sent on to reviewers who are experts in this field of research, and the editorial board makes its decisions based on the reviews of these experts. The article may be accepted as is, sent back for improvements, or rejected.

The editorial board can decide to reject an article if it does not conform to the guidelines set above.

A manuscript which has been sent back to the authors for improvements requested by the editors and/ or reviewers is reviewed again, after which the editorial board makes another decision on whether the article can be accepted for publication. The published article has the submission and publication acceptance dates set at the beginning.
The return of an article to the authors for improvement does not mean that the article has been accepted for publication. After the revised text has been received, a decision is made by the editorial board. The author must return the improved text, together with the original text and responses to all comments. The date of acceptance is the day on which the final version of the article was received by the publisher.

A revised manuscript must be sent back to the publisher a week after the authors have received the comments; if not, the article is considered a resubmission.

E-mail is used at all the stages of communication between the author, editors, publishers, and reviewers, so it is of vital importance that the authors monitor the address that they list in the article and inform the publisher of any changes in due time.

After the layout for the relevant issue of the journal is ready, the publisher sends out PDF files to the authors for a final review.

Changes other than simple corrections in the text, figures, or tables are not allowed at the final review stage. If this is necessary, the issue is resolved by the editorial board.

\section{FORMAT OF REFERENCES}

The journal uses a numeric reference system, which means that references are denoted as numbers in the text (in brackets) which refer to the number in the reference list.

For books: the last name and initials of the author, full title of the book, location of publisher, publisher, year in which the work was published, and the volume or issue and the number of pages in the book.

For periodicals: the last name and initials of the author, title of the journal, year in which the work was published, volume, issue, first and last page of the article.

Bressanelli S., Tomei L., Roussel A., et al // Proc. Natl. Acad. Sci. USA. 1999. V. 96. P.13034-13039 (If there are more than five authors). If there are less than five authors, all the authors must be listed.

References to books which have Russian translations should be accompanied with references to the original material listing the required data.

References to doctoral thesis abstracts must include the last name and initials of the author, the title of the thesis, the location in which the work was performed, and the year of completion.

References to patents must include the last names and initials of the authors, the type of the patent document (the author's rights or patent), the patent number, the name of the country that issued the document, the international invention classification index, and the year of patent issue.

The list of references should be on a separate page. The tables should be on a separate page, and figure captions should also be on a separate page.

The following e-mail addresses can be used to contact the editorial staff: vera.knorre@gmail. com, actanaturae@gmail.com, tel.: (495) 727-38-60, (495) 930-80-05 